\title{
JPSS-1 VIIRS at-launch geometric performance
}

NASA VIIRS Characterization Support Team (VCST) Geometric Calibration Group

Guoqing (Gary) Lin, SSAI/GSFC Code 619

Robert E. Wolfe, NASA/GSFC Code 619

SPIE Optics+Photonics Conference, 28 Aug - -1 Sept 2016

San Diego, California

Thursday, 1 Sept 2016 


\section{Outline}

- Spatial Responses, LSF, DFOV, MTF

- Band-to-Band Co-registration (BBR)

- Pointing (for geolocation)

- DNB Geometric Performance

- Concluding Remarks 


\section{Optical calibration}
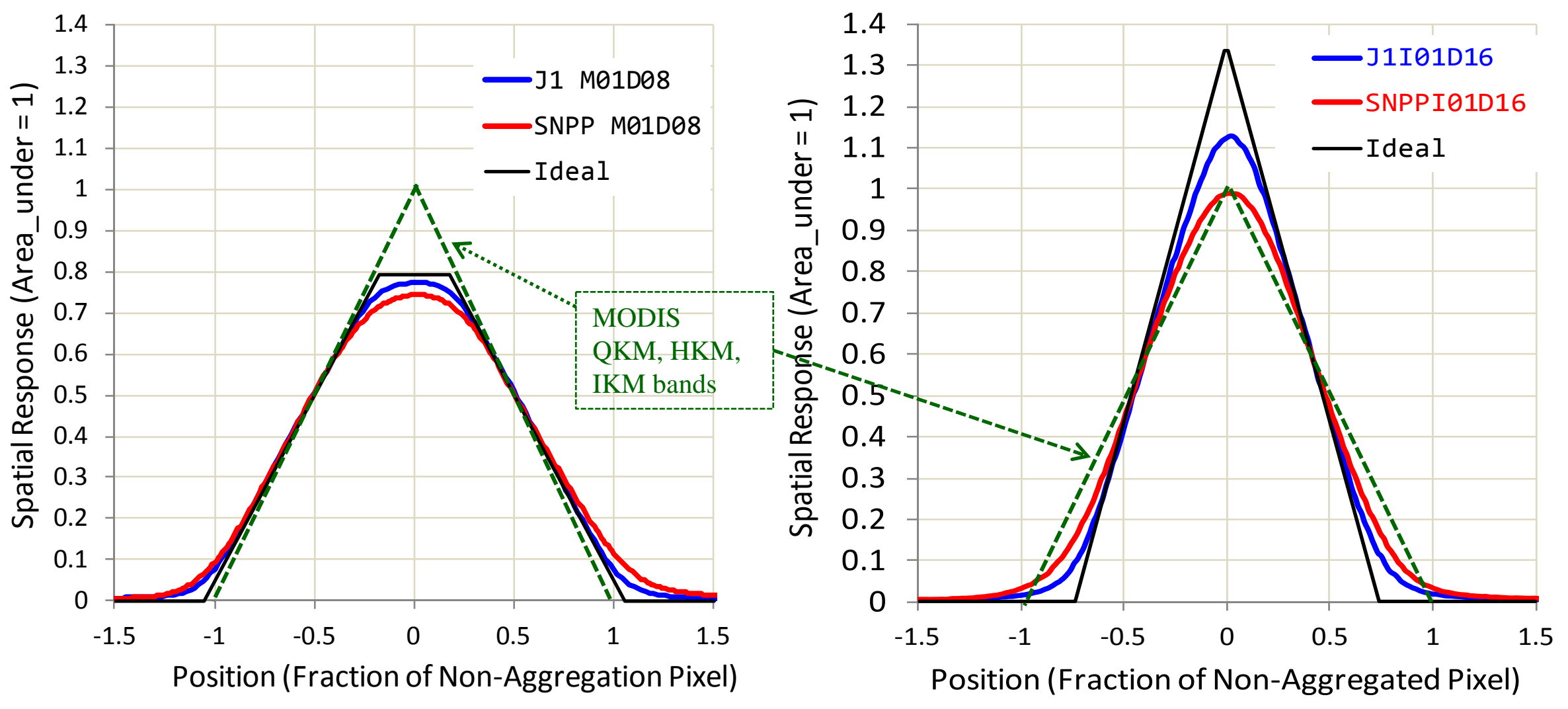

$>\mathrm{J} 1$ VIIRS has improved optical system over SNPP efforts were made to improve the mirrors and the coating for the RTA 


\section{Scan LSF -- DFOV}

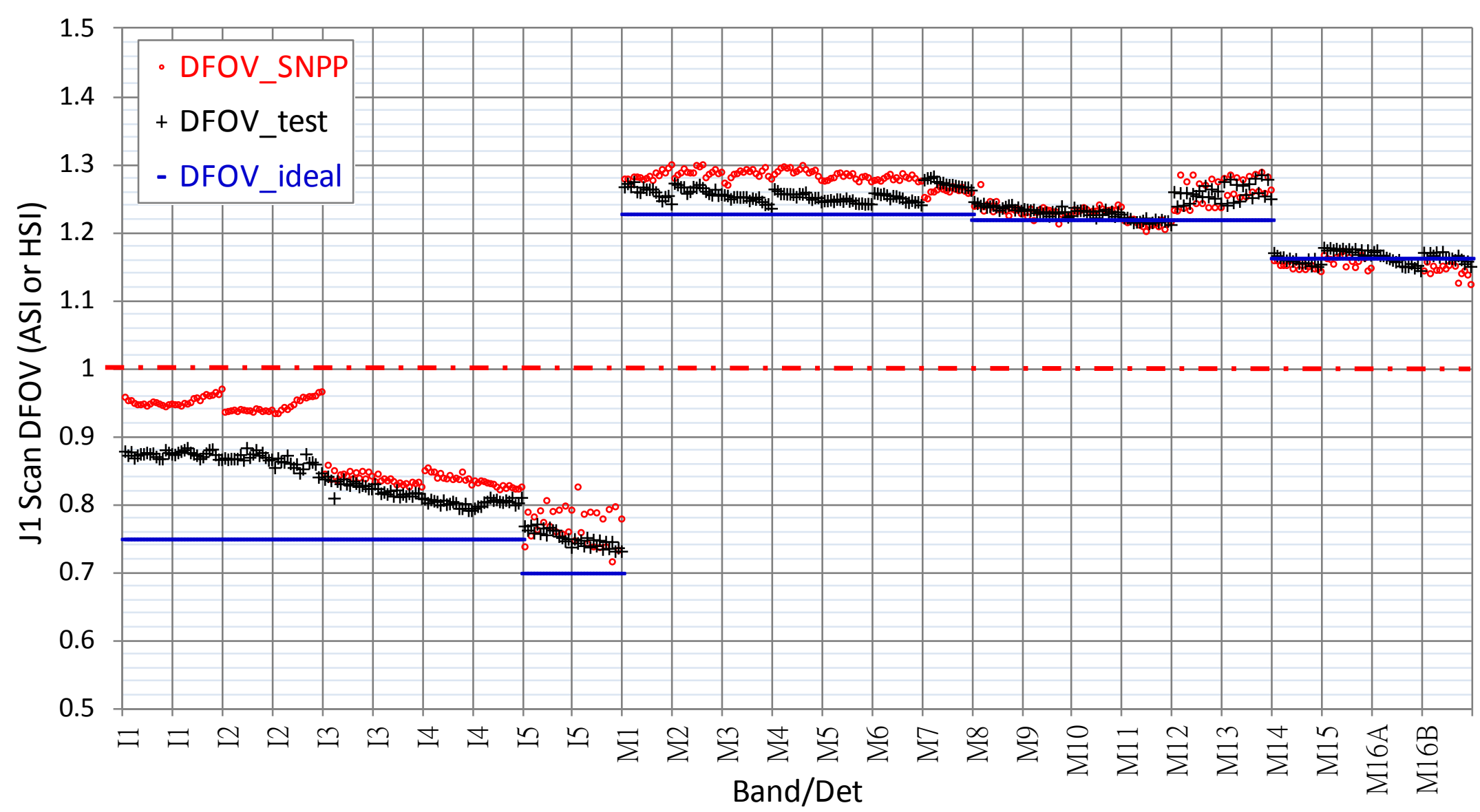

- J1 F2 optical performance is good (better than SNPP F1)

- M-Bands over-sample the earth, in the un-aggregated zones

- I-bands under-sample the earth (TOA), mostly in the un-aggregated zones

- Track direction LSFs are mostly square, IFOV = 1.0 ASI (or HSI on the ground) 


\section{Scan LSF -- MTF}

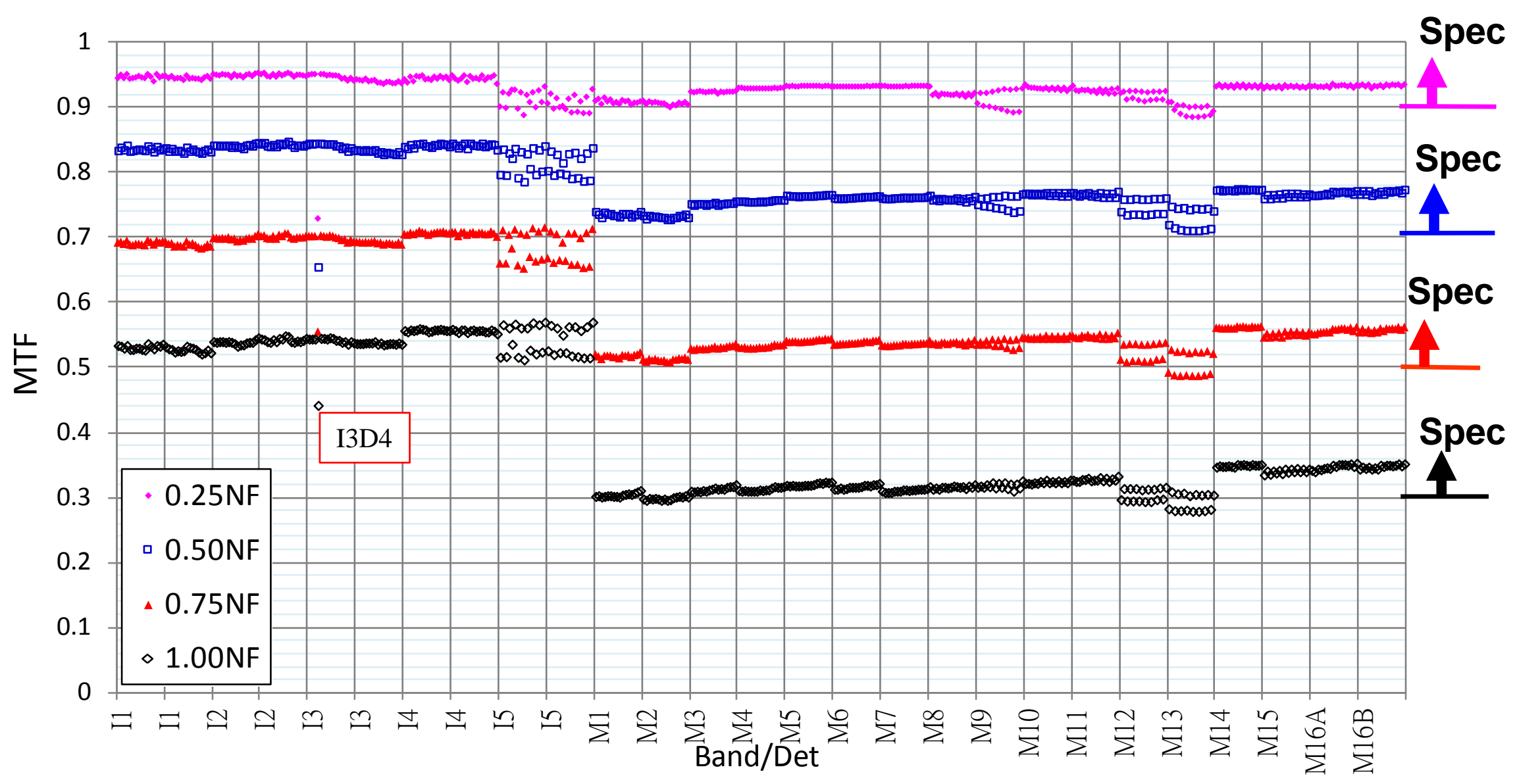

- MTF for M-bands mostly meets specification

- I-bands images are very sharp, at least at TOA (I3D4 under-performs but is still good in MTF)

- Track direction LSFs are mostly square, MTF = 0.63 at 1.00NF (Nyquist Frequency) 


\section{Scan LSF $\rightarrow$ MTF w/SNPP}

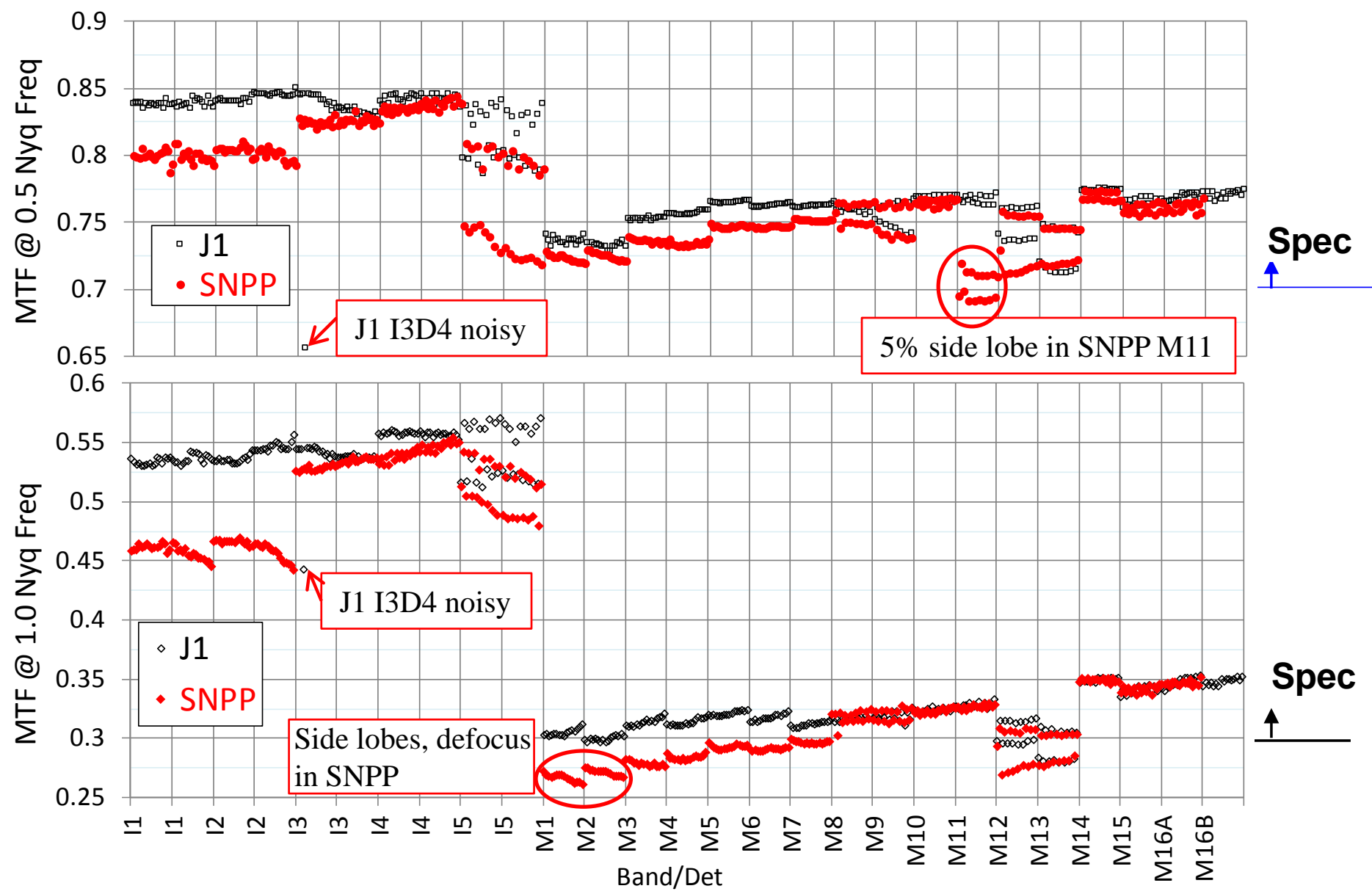

$>$ J1 MTF performs better than SNPP

$>$ Side-lobes of M11 in SNPP disappear in J1

$>$ Right focus for VisNIR FPA/bands in J1, while defocused/shorter EFL in SNPP 


\section{VIIRS Band/Detector Physical Layout}

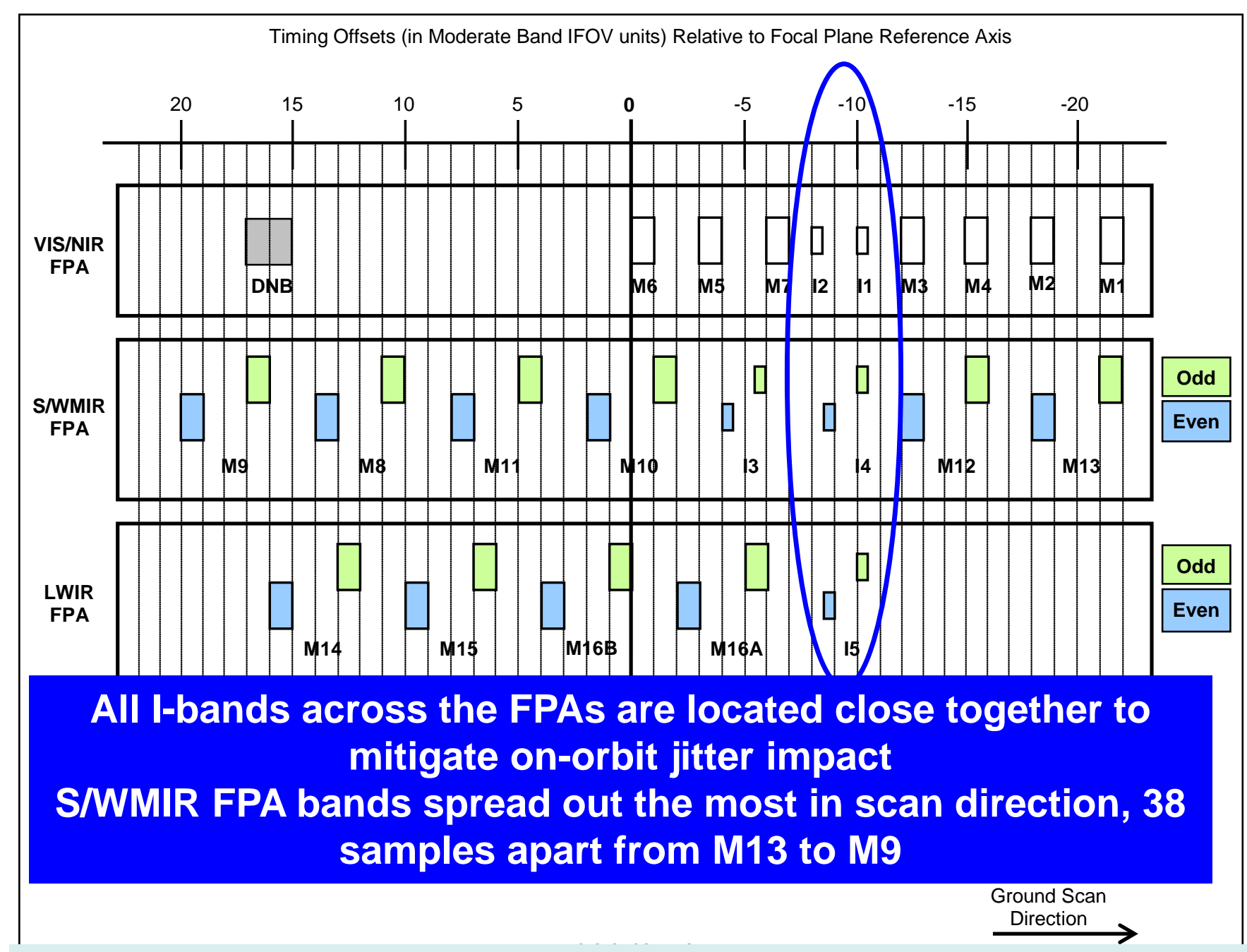

3 focal planes: VisNIR, SWMIR, LWIR; + 1 DNB (no BBR Spec)

21 bands (16 M-bands (M16A, M16B merged in space or just sent down one), 5 I-bands) 16 detectors in each $M$-band; 32 detectors in each I-band 


\section{M-band Sample Aggregation Sample Numbers Pixel Size}

\begin{tabular}{r|l} 
Sample No. & $1600(3152)$ \\
\hline HSI Scan (m) & $774(258)$ \\
\hline
\end{tabular}

\begin{tabular}{rl|l}
$1009(1377)$ & $1008(1376)$ \\
$1161(387)$ & $774(387)$ \\
893 &
\end{tabular}

\begin{tabular}{r|r}
$641(641)$ & $640(640)$ \\
\hline $1295(648)$ & $648(648)$ \\
\hline 1123 &
\end{tabular}

1(1)

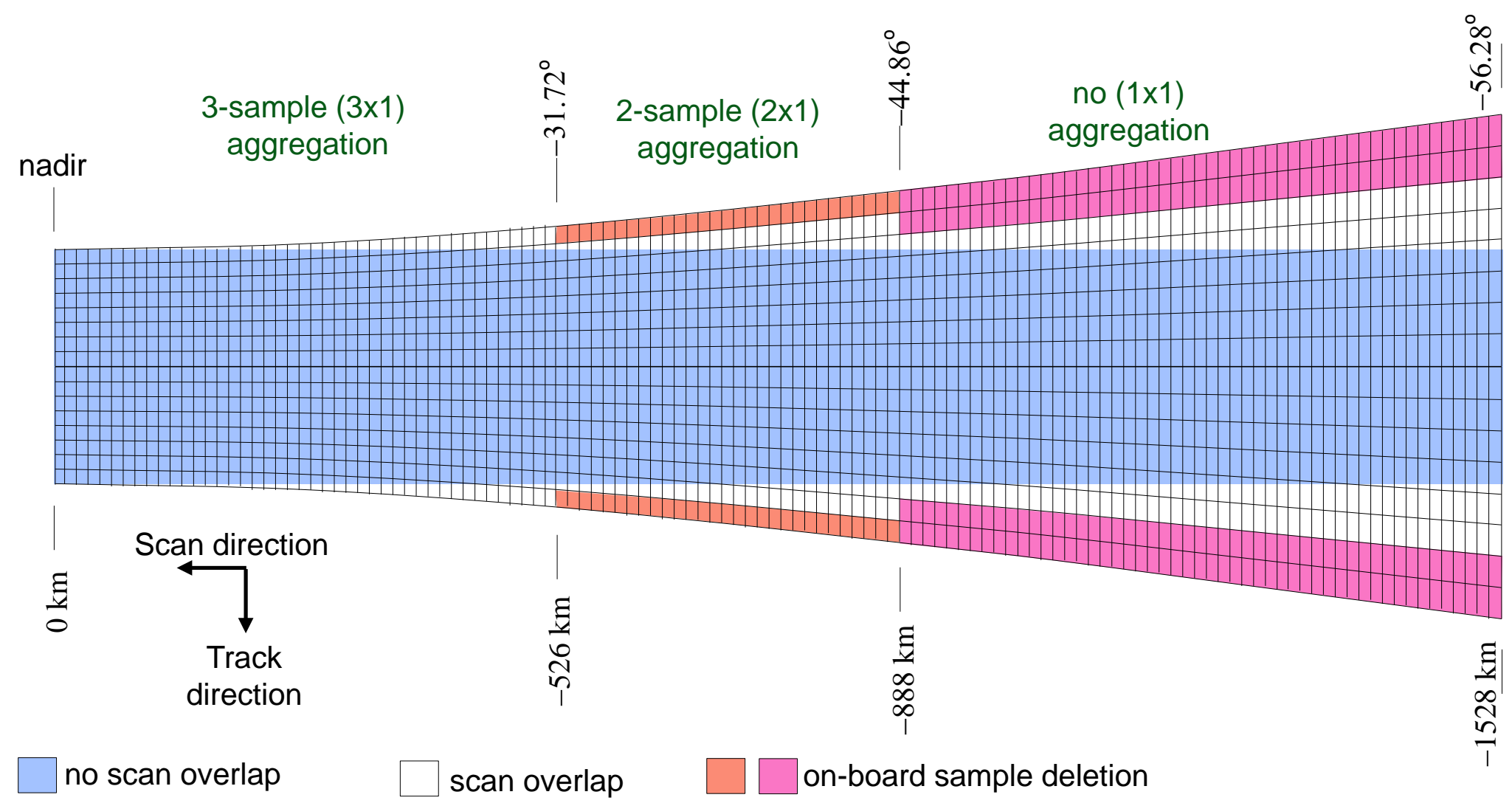

On-board sample deletion deletes $2 \mathrm{M}$-band (4 I-band) detectors in the 2 sample aggregation zone and $4 \mathrm{M}$-band (8 Iband) detectors in the no-aggregation zone. The numbers in in parentheses for the "Sample no." and "HSI Scan (m)" are for dual-gain M-bands before aggregation, SDR of which are available to the ground as intermediate products. 


\section{M-band Sample Aggregation Sample Numbers Pixel Size}

\begin{tabular}{r|lrr|rr|r|} 
Sample No. & $1600(3152)$ & $1009(1377)$ & $1008(1376)$ & $641(641)$ & $640(640)$ & $1(1)$ \\
\hline HSI Scan $(\mathrm{m})$ & $774(258)$ & $1152(384)$ & $768(384)$ & $1277(638)$ & $638(638)$ & $1600(1600)$ \\
\hline HSI Track (m) & 741 & 888 & 1113 & 1603
\end{tabular}

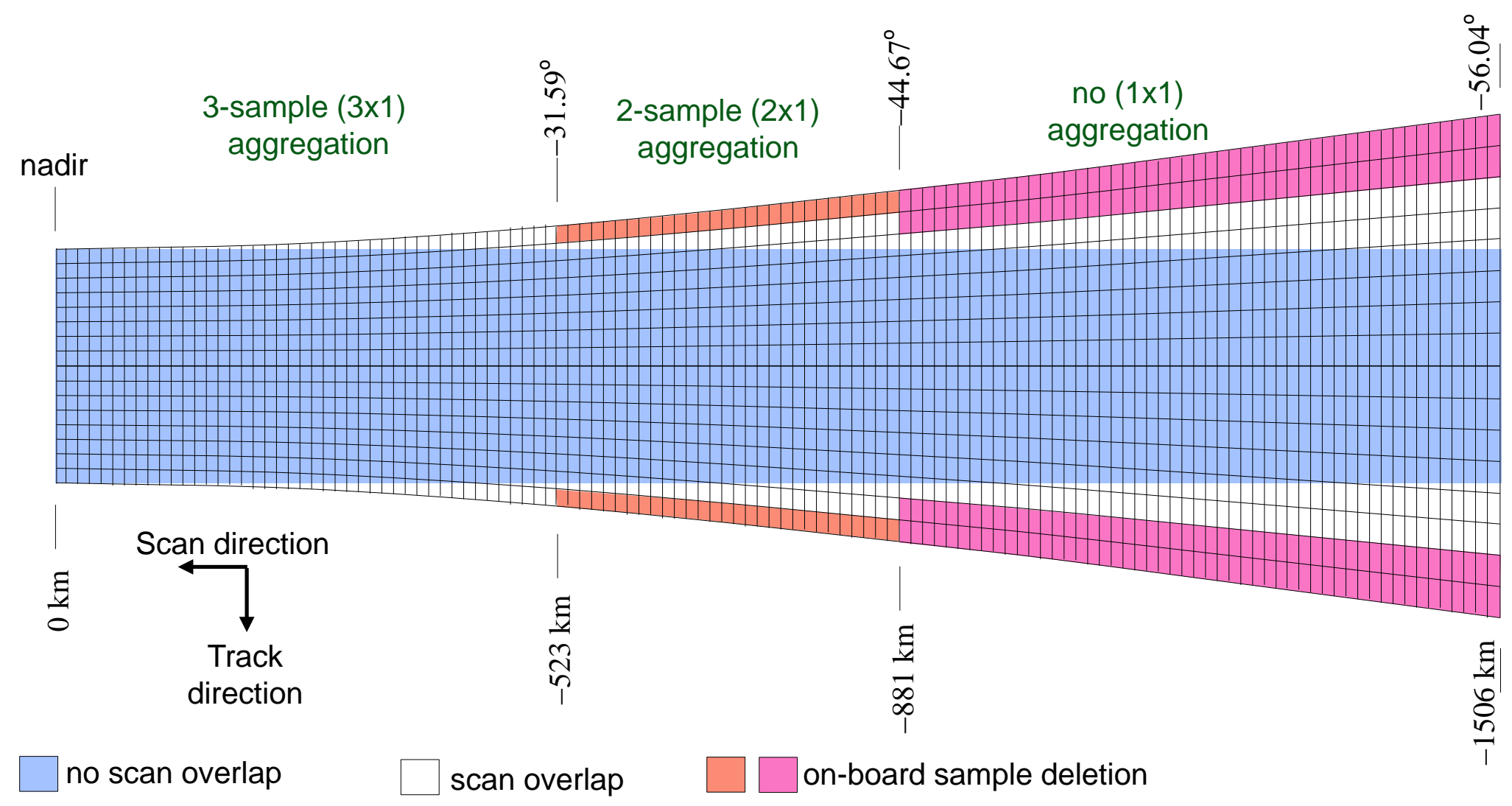

On-board sample deletion deletes $2 \mathrm{M}$-band (4 I-band) detectors in the 2 sample aggregation zone and $4 \mathrm{M}$-band (8 Iband) detectors in the no-aggregation zone. The numbers in in parentheses for the "Sample no." and "HSI Scan (m)" are for dual-gain M-bands before aggregation, SDR of which are available to the ground as intermediate products. 


\section{Track (mis-)Registration wrt I1}

SNPP

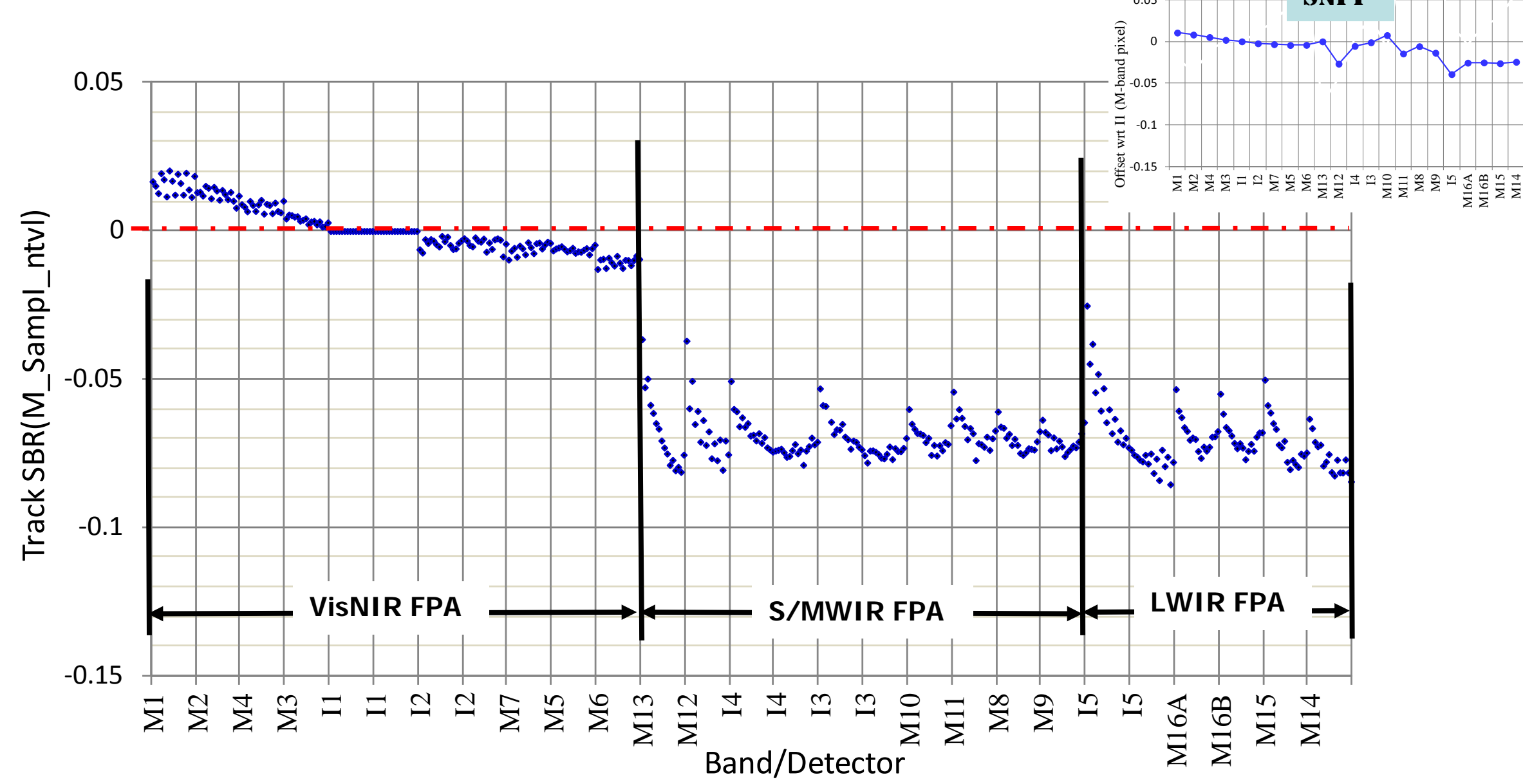

- Results obtained from tests at the nominal temperature performance plateau

- Track direction bands co-register well within each FPA

- Bands on SWMWIR and LWIR FPAs shifted from bands on VisNIR FPA, 7\% for Mbands and $\sim 14 \%$ for I-bands. Mapping uncertainties are also affected $R M S E=\sqrt{\sigma^{2}+\mu^{2}}$ 


\section{Scan (mis-)Registration wrt I1}

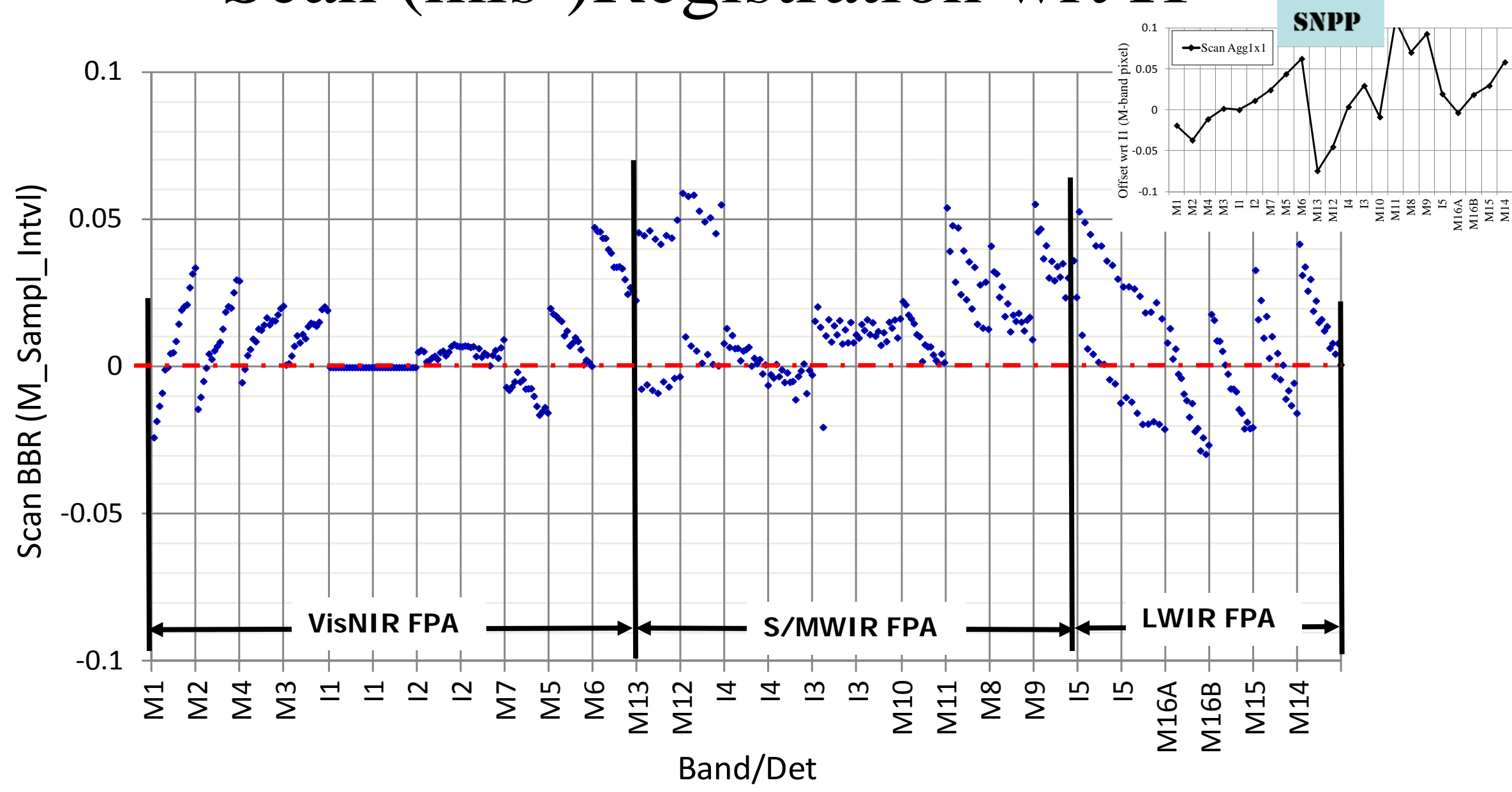

- Results obtained from tests at the nominal temperature performance plateau

- The scan rate is nominal @1.786 sec/scan or $3.517 \mathrm{rad} / \mathrm{sec}(0.4 \%$ slower than SNPP)

- Data shows for un-aggregated zones. Mis-reg in Agg2x1 and $3 \times 1$ zones is $1 / 2$ and $1 / 3$

- Mis-reg is < $-5 \%$ for M-band and < $10 \%$ for I-bands 


\section{BBR matrix- non-agg zones}

\begin{tabular}{|c|c|c|c|c|c|c|c|c|c|c|c|c|c|c|c|c|c|c|c|c|c|c|}
\hline Band & M1 & M2 & M3 & M4 & M5 & M6 & M7 & M8 & M9 & M10 & M11 & M12 & M13 & M14 & M15 & M16A & M16B & I1 & 12 & 13 & 14 & 15 \\
\hline M1 & & 0.99 & .97 & 0.98 & 0.95 & 0.92 & 0.93 & 0.88 & .86 & 0.89 & 0.87 & 0.86 & 0.87 & 0.87 & 0.87 & 0.86 & 0.86 & 0.95 & 0.95 & 0.89 & 0.88 & 0.88 \\
\hline M2 & .64 & & 98 & .98 & 95 & 93 & .94 & 89 & 87 & .89 & 88 & 87 & 88 & 88 & 87 & 87 & & 96 & 96 & 90 & 88 & 88 \\
\hline M3 & 64 & 64 & & .99 & 97 & 94 & 0.96 & .90 & 88 & 0.91 & 0.89 & 89 & 89 & 89 & 89 & 88 & & .97 & 97 & .91 & .90 & 0.90 \\
\hline M4 & & 64 & & & 97 & & 0.95 & .90 & 88 & .90 & 0.89 & .88 & 0.88 & 0.89 & 89 & 88 & & 0.97 & .97 & & 0.89 & 0.89 \\
\hline $\mathrm{M}$ & & & & & & & 0.97 & .92 & 90 & 0.93 & & & 88 & & 01 & & & & .98 & & 0.92 & 0.92 \\
\hline M6 & & & & .64 & & & .94 & & & & & & & & & & & & & & & \\
\hline M7 & & & & .64 & .80 & .04 & & & & 0.91 & & & & & & & & & & & & \\
\hline M8 & & 64 & 64 & 0.64 & 64 & .64 & 0.64 & & 98 & 0.98 & 0.98 & .96 & .96 & 0.98 & .97 & 95 & & 0.90 & .91 & 0.98 & 0.97 & 0.98 \\
\hline M9 & .64 & 64 & 64 & 0.64 & 64 & 0.64 & 0.64 & .64 & & 0.97 & 0.98 & .96 & .94 & 0.97 & .95 & .94 & 95 & 0.88 & .89 & .96 & 0.95 & 0.96 \\
\hline M10 & 0.64 & .64 & .64 & 0.64 & .64 & 0.64 & 0.64 & 0.64 & 0.64 & & 0.96 & 0.94 & 0.95 & 0.98 & 0.98 & 0.97 & 0.98 & 0.92 & 0.92 & 0.99 & 0.98 & 0.98 \\
\hline M11 & 064 & .64 & 0.70 & 0.64 & 0.70 & 0.64 & 0.64 & 0.64 & 0.64 & 0.64 & & 0.97 & 0.96 & 0.97 & 0.95 & 0.94 & 0.95 & 0.89 & 0.90 & 0.96 & 0.95 & 0.96 \\
\hline M12 & 061 & .64 & 0.64 & 0.64 & .64 & 0.64 & 0.64 & 0.64 & 0.80 & 0.64 & & & 0.98 & 0.95 & 0.94 & 0.92 & & 0.88 & & & 0.93 & 0.94 \\
\hline M13 & & 64 & & 0.64 & 64 & 64 & 0.64 & .64 & 0.80 & 0.64 & 0.64 & 0.80 & & & .95 & .92 & & 0.88 & & 0.95 & 0.94 & 0.95 \\
\hline M1 & & & & 0.64 & 64 & 64 & 0.64 & 64 & 80 & .64 & 64 & .80 & .80 & & & & & 0.89 & & .97 & 0.96 & 0.97 \\
\hline M15 & & & & 0.64 & 64 & 64 & 0.64 & 64 & 80 & & & 80 & 80 & & & 0.97 & & & & & .98 & 0.98 \\
\hline M16A & .64 & 64 & 64 & 0.64 & 64 & 64 & 0.64 & 0.64 & 80 & 64 & 0.64 & .80 & 80 & 0.80 & & & & .90 & .90 & 0.96 & 0.97 & 0.96 \\
\hline & 61 & 64 & & 0.64 & 64 & & 0.64 & 64 & 80 & 64 & & & & & & & & 0.91 & & 96 & 0.98 & 0.97 \\
\hline I1 & & & & & & & 0.64 & & & & & & & & & & & & & & & \\
\hline 12 & & & & 0.64 & .64 & 0.64 & 0.64 & & 0.64 & 0.64 & & 0.64 & & & & & & 0.80 & & & 0.83 & \\
\hline 13 & & 64 & & 0.64 & 0.64 & 0.64 & 0.64 & 0.64 & 0.64 & 0.64 & 0.64 & 0.64 & 0.64 & 0.64 & 0.64 & 0.64 & & 0.80 & 0.80 & & & \\
\hline 14 & 0.64 & 0.64 & 0.64 & 0.64 & 0.64 & 0.64 & 0.64 & 0.64 & 0.64 & 0.64 & 0.64 & 0.64 & 0.64 & 0.64 & 0.64 & 0.64 & 0.64 & 0.80 & 0.80 & & & 0.89 \\
\hline 15 & 0.64 & .64 & 0.64 & 0.64 & 0.64 & 0.64 & 0.64 & 0.64 & 0.64 & 0.64 & 0.64 & 0.64 & 0.64 & 0.64 & 0.64 & 0.64 & 0.64 & 0.80 & & & 0.80 & \\
\hline
\end{tabular}

- None out of spec at the ground test level

Barely made it

- On-orbit jitter should have little impact since I-bands are located close together with very short time delay for co-reg. 


\section{BBR matrix-2x1-agg zones}

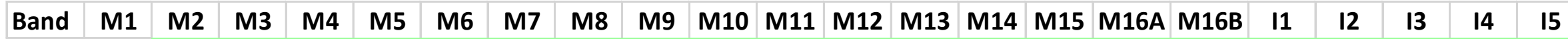

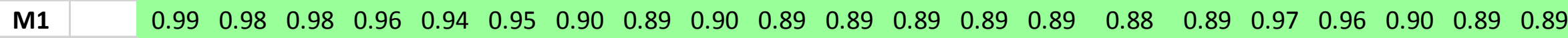
\begin{tabular}{l|l|lllllllllllllllllllll} 
M2 & 0.64 & & 0.99 & 0.99 & 0.97 & 0.95 & 0.96 & 0.90 & 0.89 & 0.90 & 0.90 & 0.89 & 0.89 & 0.90 & 0.89 & 0.89 & 0.89 & 0.97 & 0.97 & 0.91 & 0.90 & 0.90
\end{tabular} \begin{tabular}{l|l|l|llllllllllllll} 
M3 & 0.64 & 0.64 & & 0.99 & 0.98 & 0.96 & 0.97 & 0.91 & 0.91 & 0.92 & 0.91 & 0.91 & 0.90 & 0.91 & 0.91
\end{tabular} \begin{tabular}{l|l|l|l|l|l|l|l|l|lllllll} 
M4 & 0.64 & 0.64 & 0.64 & & 0.98 & 0.96 & 0.97 & 0.91 & 0.90 & 0.91 & 0.91 & 0.90 & 0.90 & 0.91 & 0.90
\end{tabular} \begin{tabular}{l|l|l|l|l|l|l|l|l|l|l|l|l|l|l|l} 
M5 & 0.64 & 0.64 & 0.70 & 0.64 & & 0.98 & 0.99 & 0.93 & 0.92 & 0.93 & 0.92 & 0.91 & 0.90 & 0.92 & 0.92 \\
\hline
\end{tabular} \begin{tabular}{l|l|l|l|l|l|l|l|l|l|l|l|l|l|l|l|l}
\hline & 0.96 & 0.64 & 0.64 & 0.64 & 0.64 & 0.64 & & 0.97 & 0.93 & 0.93 & 0.92 & 0.93 & 0.92 & 0.91 & 0.92 & 0.91 \\
\hline
\end{tabular}

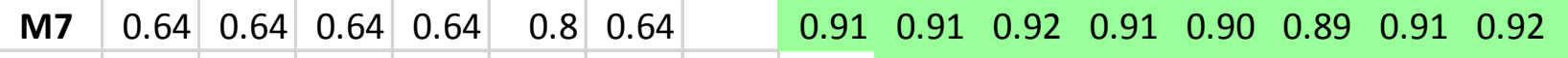

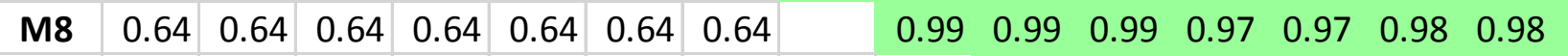

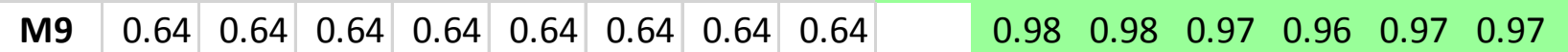

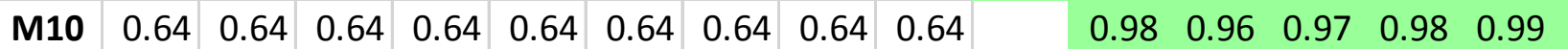
\begin{tabular}{|l|l|l|l|l|l|l|l|l|l|l|l|l|l|l|}
\hline M11 & 0.64 & 0.64 & 0.70 & 0.64 & 0.70 & 0.64 & 0.64 & 0.64 & 0.64 & 0.64 & 0.98 & 0.97 & 0.98 & 0.97 \\
\hline
\end{tabular} \begin{tabular}{|l|l|l|l|l|l|l|l|l|l|l|l|l|l|l|}
\hline M12 & 0.64 & 0.64 & 0.64 & 0.64 & 0.64 & 0.64 & 0.64 & 0.64 & 0.80 & 0.64 & 0.64 & 0.98 & 0.96 & 0.96 \\
\hline
\end{tabular} \begin{tabular}{|l|l|l|l|l|l|l|l|l|l|l|l|l|l|l|}
\hline M13 & 0.64 & 0.64 & 0.64 & 0.64 & 0.64 & 0.64 & 0.64 & 0.64 & 0.80 & 0.64 & 0.64 & 0.80 & 0.97 & 0.97 \\
\hline
\end{tabular} \begin{tabular}{|l|l|l|l|l|l|l|l|l|l|l|l|l|l|l|}
\hline M14 & 0.64 & 0.64 & 0.64 & 0.64 & 0.64 & 0.64 & 0.64 & 0.64 & 0.80 & 0.64 & 0.64 & 0.80 & 0.80 & 0.98 \\
\hline
\end{tabular} \begin{tabular}{|l|l|l|l|l|l|l|l|l|l|l|l|l|l|l|l|l|l|l|}
\hline M15 & 0.64 & 0.64 & 0.64 & 0.64 & 0.64 & 0.64 & 0.64 & 0.64 & 0.80 & 0.64 & 0.64 & 0.80 & 0.80 & 0.80 & 0.98 \\
\hline
\end{tabular} \begin{tabular}{|l|l|l|l|l|l|l|l|l|l|l|l|l|l|l|l|}
\hline M16A & 0.64 & 0.64 & 0.64 & 0.64 & 0.64 & 0.64 & 0.64 & 0.64 & 0.80 & 0.64 & 0.64 & 0.80 & 0.80 & 0.80 & 0.80 \\
\hline
\end{tabular} \begin{tabular}{|l|l|l|l|l|l|l|l|l|l|l|l|l|l|l|l|}
\hline M16B & 0.64 & 0.64 & 0.64 & 0.64 & 0.64 & 0.64 & 0.64 & 0.64 & 0.80 & 0.64 & 0.64 & 0.80 & 0.80 & 0.80 & 0.80 \\
\hline
\end{tabular} \begin{tabular}{|l|l|l|l|l|l|l|l|l|l|l|l|l|l|l|l|}
\hline I1 & 0.64 & 0.64 & 0.64 & 0.64 & 0.64 & 0.64 & 0.64 & 0.64 & 0.64 & 0.64 & 0.64 & 0.64 & 0.64 & 0.64 & 0.64 \\
\hline
\end{tabular} \begin{tabular}{|l|l|l|l|l|l|l|l|l|l|l|l|l|l|l|l|}
\hline 12 & 0.64 & 0.64 & 0.64 & 0.64 & 0.64 & 0.64 & 0.64 & 0.64 & 0.64 & 0.64 & 0.64 & 0.64 & 0.64 & 0.64 & 0.64 \\
\hline
\end{tabular} \begin{tabular}{|l|l|l|l|l|l|l|l|l|l|l|l|l|l|l|l|}
\hline I3 & 0.64 & 0.64 & 0.64 & 0.64 & 0.64 & 0.64 & 0.64 & 0.64 & 0.64 & 0.64 & 0.64 & 0.64 & 0.64 & 0.64 & 0.64 \\
\hline
\end{tabular} \begin{tabular}{|l|l|l|l|l|l|l|l|l|l|l|l|l|l|l|l|}
\hline 14 & 0.64 & 0.64 & 0.64 & 0.64 & 0.64 & 0.64 & 0.64 & 0.64 & 0.64 & 0.64 & 0.64 & 0.64 & 0.64 & 0.64 & 0.64 \\
\hline
\end{tabular} \begin{tabular}{|l|l|l|l|l|l|l|l|l|l|l|l|l|l|l|l|}
\hline $\mathbf{1 5}$ & 0.64 & 0.64 & 0.64 & 0.64 & 0.64 & 0.64 & 0.64 & 0.64 & 0.64 & 0.64 & 0.64 & 0.64 & 0.64 & 0.64 & 0.64 \\
\hline
\end{tabular}

- None out of spec at the ground test level $\begin{array}{lllllll}0.90 & 0.90 & 0.99 & 0.98 & 0.92 & 0.91 & 0.91\end{array}$ $\begin{array}{lllllll}0.90 & 0.90 & 0.98 & 0.98 & 0.91 & 0.91 & 0.90\end{array}$ $\begin{array}{llllllll}0.92 & 0.92 & 0.98 & 0.99 & 0.93 & 0.93 & 0.93\end{array}$ $\begin{array}{llllllll}0.91 & 0.91 & 0.96 & 0.97 & 0.92 & 0.92 & 0.92\end{array}$ $\begin{array}{lllllll}0.93 & 0.93 & 0.99 & 0.99 & 0.92 & 0.92 & 0.92\end{array}$ $\begin{array}{llllllll}0.97 & 0.98 & 0.92 & 0.92 & 0.99 & 0.98 & 0.98\end{array}$ $\begin{array}{llllllll}0.97 & 0.97 & 0.91 & 0.91 & 0.98 & 0.97 & 0.97\end{array}$ $\begin{array}{llllllll}0.98 & 0.98 & 0.92 & 0.93 & 0.99 & 0.99 & 0.98\end{array}$ $\begin{array}{llllllll}0.97 & 0.97 & 0.91 & 0.91 & 0.98 & 0.97 & 0.98\end{array}$ $\begin{array}{llllllll}0.96 & 0.96 & 0.90 & 0.91 & 0.96 & 0.96 & 0.97\end{array}$ $\begin{array}{llllllll}0.95 & 0.95 & 0.90 & 0.90 & 0.97 & 0.96 & 0.97\end{array}$ $\begin{array}{llllllll}0.97 & 0.97 & 0.91 & 0.92 & 0.98 & 0.98 & 0.98\end{array}$ $\begin{array}{llllllll}0.98 & 0.98 & 0.92 & 0.92 & 0.98 & 0.99 & 0.98\end{array}$ 0.80 $\begin{array}{lllllll}0.99 & 0.91 & 0.92 & 0.97 & 0.98 & 0.98\end{array}$ \begin{tabular}{|l|l|l|l|l|l|l|l|l|l|l|}
0.80 & & 0.92 & 0.92 & 0.98 & 0.98 & 0.98 \\
\hline 0.64 & 0.64 & & 0.98 & 0.83 & 0.84 & 0.81 \\
\hline
\end{tabular} \begin{tabular}{l|l|l|l|l|l|l}
0.64 & 0.64 & 0.80 & 0.84 & 0.84 & 0.83
\end{tabular} \begin{tabular}{|l|l|l|l|l|l|l|}
\hline 0.64 & 0.64 & 0.80 & 0.80 & & 0.97 & 0.92 \\
\hline 0.64 & 0.64 & 0.80 & 0.80 & 0.80 & 0.92 \\
\hline
\end{tabular} 0.92 0.64

- On-orbit jitter should have little impact since I-bands are located close together with very short time delay for co-reg. 


\section{BBR matrix-3x1-agg zones}

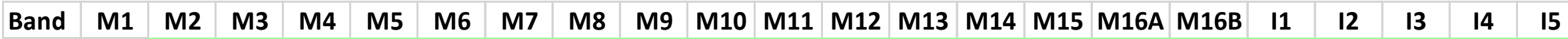

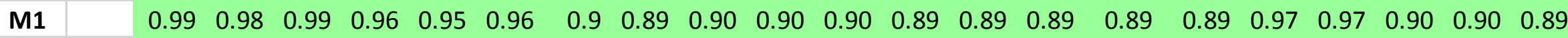
$\begin{array}{llllllllllllllllllllllll}\text { M2 } & 0.64 & & 0.99 & 0.99 & 0.97 & 0.95 & 0.97 & 0.91 & 0.90 & 0.91 & 0.90 & 0.90 & 0.90 & 0.89 & 0.90 & 0.90 & 0.90 & 0.98 & 0.97 & 0.91 & 0.90 & 0.90\end{array}$ \begin{tabular}{l|l|l|llllllllllllllllllll} 
M3 & 0.64 & 0.64 & & 0.99 & 0.98 & 0.97 & 0.98 & 0.92 & 0.91 & 0.92 & 0.91 & 0.91 & 0.91 & 0.91 & 0.91 & 0.91 & 0.91 & 0.99 & 0.99 & 0.92 & 0.91 & 0.91
\end{tabular} \begin{tabular}{l|l|l|l|l|l|l|l|l|lllllll} 
M4 & 0.64 & 0.64 & 0.64 & & 0.98 & 0.96 & 0.97 & 0.91 & 0.91 & 0.91 & 0.91 & 0.91 & 0.90 & 0.90 & 0.90
\end{tabular} \begin{tabular}{l|l|l|l|l|l|l|l|l|l|l|l|l|l|l|l|l|l}
\hline M5 & 0.64 & 0.64 & 0.70 & 0.64 & & 0.98 & 0.99 & 0.93 & 0.92 & 0.93 & 0.92 & 0.92 & 0.91 & 0.92 & 0.92 \\
\hline
\end{tabular} $\begin{array}{lllllll}0.90 & 0.91 & 0.98 & 0.98 & 0.91 & 0.91 & 0.90\end{array}$ \begin{tabular}{l|l|l|l|l|l|l|l|l|l|l|l|l|l|l|l|l|l|l|l}
\hline M6 & 0.64 & 0.64 & 0.64 & 0.64 & 0.64 & 0.98 & 0.93 & 0.93 & 0.92 & 0.93 & 0.92 & 0.92 & 0.92 & 0.92 \\
\hline
\end{tabular} \begin{tabular}{l|l|l|l|l|l|l|l|l|l|l|l|l|l|l|l|l|l|}
\hline M7 & 0.64 & 0.64 & 0.64 & 0.64 & 0.80 & 0.64 & 0.92 & 0.92 & 0.92 & 0.91 & 0.91 & 0.90 & 0.91 & 0.92 \\
\hline
\end{tabular}

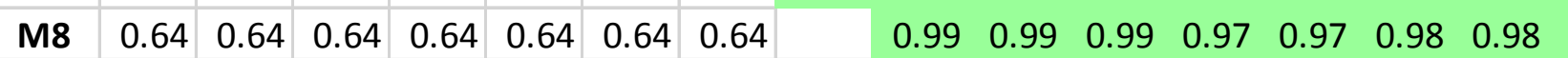
\begin{tabular}{|l|l|l|l|l|l|l|l|l|l|l|l|l|l|l|l|}
\hline M9 & 0.64 & 0.64 & 0.64 & 0.64 & 0.64 & 0.64 & 0.64 & 0.64 & & 0.98 & 0.99 & 0.97 & 0.97 & 0.97 & 0.98 \\
\hline
\end{tabular} \begin{tabular}{|l|l|l|l|l|l|l|l|l|l|l|l|l|l|l|l|l|}
\hline M10 & 0.64 & 0.64 & 0.64 & 0.64 & 0.64 & 0.64 & 0.64 & 0.64 & 0.64 & & 0.98 & 0.96 & 0.97 & 0.98 & 0.98 \\
\hline
\end{tabular} \begin{tabular}{|l|l|l|l|l|l|l|l|l|l|l|l|l|l|l|l|}
\hline M11 & 0.64 & 0.64 & 0.70 & 0.64 & 0.70 & 0.64 & 0.64 & 0.64 & 0.64 & 0.64 & 0.98 & 0.97 & 0.98 & 0.98 \\
\hline
\end{tabular} \begin{tabular}{|l|l|l|l|l|l|l|l|l|l|l|l|l|l|l|l|}
\hline M12 & 0.64 & 0.64 & 0.64 & 0.64 & 0.64 & 0.64 & 0.64 & 0.64 & 0.80 & 0.64 & 0.64 & 0.99 & 0.97 & 0.97 \\
\hline
\end{tabular} \begin{tabular}{|l|l|l|l|l|l|l|l|l|l|l|l|l|l|l|l|}
\hline M13 & 0.64 & 0.64 & 0.64 & 0.64 & 0.64 & 0.64 & 0.64 & 0.64 & 0.80 & 0.64 & 0.64 & 0.80 & 0.97 & 0.98 \\
\hline
\end{tabular} \begin{tabular}{|l|l|l|l|l|l|l|l|l|l|l|l|l|l|l|}
\hline M14 & 0.64 & 0.64 & 0.64 & 0.64 & 0.64 & 0.64 & 0.64 & 0.64 & 0.80 & 0.64 & 0.64 & 0.80 & 0.80 & 0.98 \\
\hline
\end{tabular} \begin{tabular}{|l|l|l|l|l|l|l|l|l|l|l|l|l|l|l|l|l|l|l|}
\hline M15 & 0.64 & 0.64 & 0.64 & 0.64 & 0.64 & 0.64 & 0.64 & 0.64 & 0.80 & 0.64 & 0.64 & 0.80 & 0.80 & 0.80 & \\
\hline
\end{tabular} \begin{tabular}{|l|l|l|l|l|l|l|l|l|l|l|l|l|l|l|l|}
\hline M16A & 0.64 & 0.64 & 0.64 & 0.64 & 0.64 & 0.64 & 0.64 & 0.64 & 0.80 & 0.64 & 0.64 & 0.80 & 0.80 & 0.80 & 0.80 \\
\hline
\end{tabular} \begin{tabular}{|l|l|l|l|l|l|l|l|l|l|l|l|l|l|l|l|}
\hline M16B & 0.64 & 0.64 & 0.64 & 0.64 & 0.64 & 0.64 & 0.64 & 0.64 & 0.80 & 0.64 & 0.64 & 0.80 & 0.80 & 0.80 & 0.80 \\
\hline
\end{tabular}

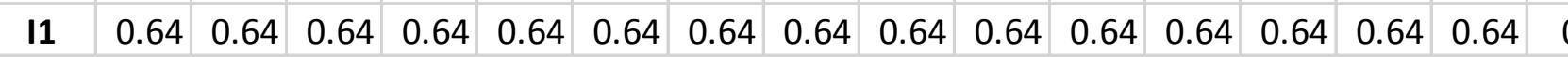

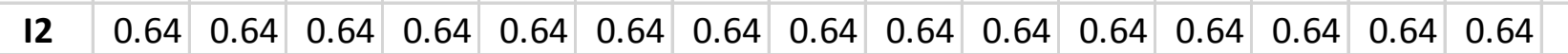

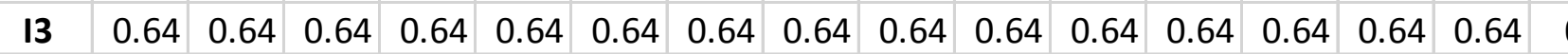
\begin{tabular}{|l|l|l|l|l|l|l|l|l|l|l|l|l|l|l|l|}
\hline 14 & 0.64 & 0.64 & 0.64 & 0.64 & 0.64 & 0.64 & 0.64 & 0.64 & 0.64 & 0.64 & 0.64 & 0.64 & 0.64 & 0.64 & 0.64 \\
\hline
\end{tabular} \begin{tabular}{|l|l|l|l|l|l|l|l|l|l|l|l|l|l|l|l|}
\hline $\mathbf{1 5}$ & 0.64 & 0.64 & 0.64 & 0.64 & 0.64 & 0.64 & 0.64 & 0.64 & 0.64 & 0.64 & 0.64 & 0.64 & 0.64 & 0.64 & 0.64 \\
\hline
\end{tabular}

- None out of spec at the ground test level $\begin{array}{lllllll}0.92 & 0.92 & 0.99 & 0.99 & 0.93 & 0.93 & 0.92\end{array}$ $\begin{array}{lllllll}0.92 & 0.92 & 0.97 & 0.98 & 0.93 & 0.92 & 0.92\end{array}$ $\begin{array}{lllllll}0.93 & 0.93 & 0.99 & 0.99 & 0.92 & 0.92 & 0.92\end{array}$ $\begin{array}{llllllll}0.98 & 0.98 & 0.92 & 0.92 & 0.99 & 0.98 & 0.97\end{array}$ $\begin{array}{llllllll}0.97 & 0.98 & 0.91 & 0.92 & 0.98 & 0.98 & 0.96\end{array}$ $\begin{array}{llllllll}0.99 & 0.99 & 0.92 & 0.93 & 0.99 & 0.99 & 0.97\end{array}$ $\begin{array}{llllllll}0.98 & 0.98 & 0.91 & 0.92 & 0.98 & 0.98 & 0.97\end{array}$ $\begin{array}{llllllll}0.97 & 0.97 & 0.91 & 0.92 & 0.97 & 0.97 & 0.97\end{array}$ $\begin{array}{llllllll}0.96 & 0.96 & 0.90 & 0.91 & 0.97 & 0.97 & 0.98\end{array}$ $\begin{array}{llllllll}0.97 & 0.98 & 0.91 & 0.92 & 0.98 & 0.98 & 0.97\end{array}$ $\begin{array}{llllllll}0.99 & 0.99 & 0.92 & 0.92 & 0.99 & 0.99 & 0.98\end{array}$ $\begin{array}{lllllll}0.99 & 0.92 & 0.92 & 0.98 & 0.99 & 0.97\end{array}$ \begin{tabular}{l|llllll}
0.80 & & 0.92 & 0.92 & 0.98 & 0.99 & 0.98 \\
\hline
\end{tabular} \begin{tabular}{|l|l|l|l|l|l|l|}
0.64 & 0.64 & & 0.98 & 0.84 & 0.84 & 0.82 \\
\hline
\end{tabular} \begin{tabular}{l|l|l|l|l|l|l|}
\hline 0.64 & 0.64 & 0.80 & & 0.85 & 0.85 & 0.83 \\
\hline
\end{tabular} \begin{tabular}{|l|l|l|l|}
\hline 0.64 & 0.64 & 0.80 & 0.80 \\
\hline
\end{tabular} \begin{tabular}{|l|l|l|l|l|}
0.64 & 0.64 & 0.80 & 0.80 & 0.80 \\
\hline
\end{tabular} 0.970 .92 \begin{tabular}{|l|l|l|l|l|l|}
0.64 & 0.64 & 0.80 & $0.80 / 0.80$ & 0.80 \\
\hline
\end{tabular}

- On-orbit jitter should have little impact since I-bands are located close together with

Improved a little, but not much very short time delay for co-reg. 


\section{Pointing (for geolocation)}

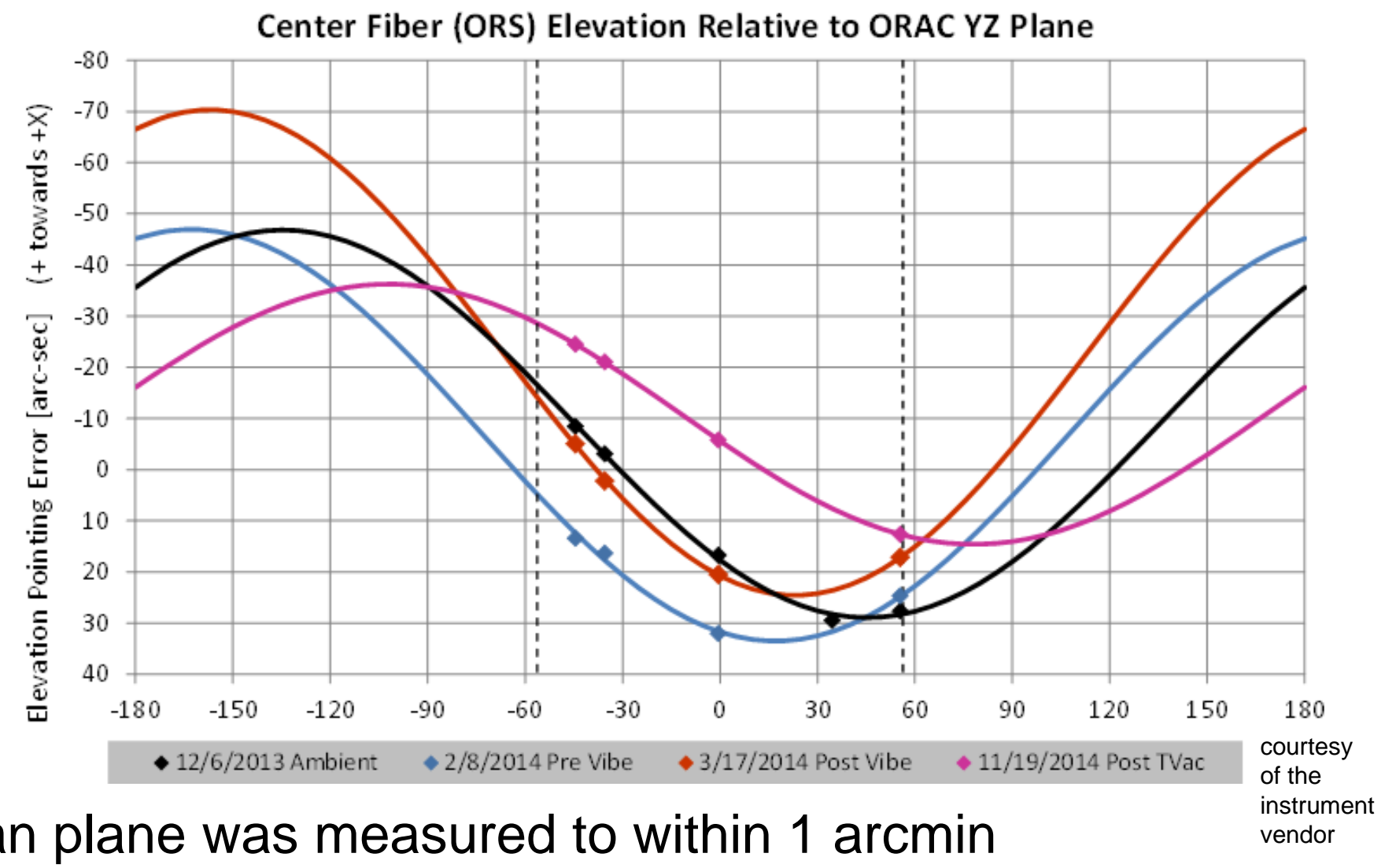

- Scan plane was measured to within 1 arcmin

- Instrument mounting ( within 1 arcmin post-vibe), launch will add to the variation

- On-orbit geolocation CalVal will remove biases and sub-pixel accuracy is expected for $\mathrm{M}$ - \& I-bands 


\section{Concluding Remarks}

- J1 VIIRS has good optical performance (better than SNPP)

- J1 VIIRS scan rate is nominal @1.786 sec/scan or 3.517 $\mathrm{rad} / \mathrm{sec}($

SNPP VIIRS is @ 1.779 sec/scan or

$3.531 \mathrm{rad} / \mathrm{sec})$

- J1 VIIRS BBR aligns well in scan direction

- However, in the track direction, bands (I3-5, M8-16) on the CFPAs are shifted from bands (I1-2, M1-7) on VisNIR FPA, 7\% for M-bands and $14 \%$ for I-bands. Mapping uncertainties will be affected

- J1 VIIRS pointing was measured. On-orbit geolocation CalVal will remove biases and sub-pixel accuracy is expected for M\& I-bands, as was done for that of SNPP VIIRS

- J1 DNB geometry is different than that of SNPP VIIRS 


\section{Backup}




\section{Image Resolution Specifications - FOVs}

- Scan Dynamic Field of View (DFOV), including integration drag

- = Full Width Half Maximum (FWHM) of Line Spread Function (LSF)

- I-bands, original Spec (actual dominant by integration_drag \& EFL)

- I1, I2: 114 (116) $\mu \mathrm{rad}$

- I3: 108 (116) $\mu \mathrm{rad}$

- 14: $109(116) \mu \mathrm{rad}$

$\pm 10 \%$ for spec

- 15: $102(109) \mu \mathrm{rad}$

- M-bands: original Spec (actual dominant by detector_size \& EFL)

- M1 to M11: 382 (381) $\mu \mathrm{rad}$

- M12, M13: 379 (378) $\mu \mathrm{rad}$

- M14, M15: 362 (361) $\mu \mathrm{rad}$

- M16: $364(361) \mu \mathrm{rad}$

$\pm 5 \%$ for spec

- Track IFOV, without integration drag

- Given by FWHM of LSF curve, mostly square

- I-bands: IFOV $=445.5 \mu \mathrm{rad} \pm 5 \%$

- M-bands: IFOV $=891 \mu \mathrm{rad} \pm 5 \%$

- Note: angular sampling interval (ASI) (and horizontal samplntvl (HSI)) at nadir w/ avg Alt=838.8 km

- I-bands scan ASI = $155.21 \mu \mathrm{rad}$ (130 m @ nadir) ->3 ASIs = 465.6 $\mu \mathrm{rad}$ (391 m @ nadir)

- I-bands track ASI $\quad=445.5 \mu \mathrm{rad}$ (381 $\mathrm{m} @$ nadir)

- M-bands scan ASI = $310.42 \mu \mathrm{rad}$ (260 m @ nadir) ->3 ASIs =931.3 $\mu \mathrm{rad}$ (790 m @ nadir)

- M-bands track ASI

$=891 \mu \mathrm{rad}$ (762 $\mathrm{m} @$ nadir) 


\section{DNB Geometric Performance}

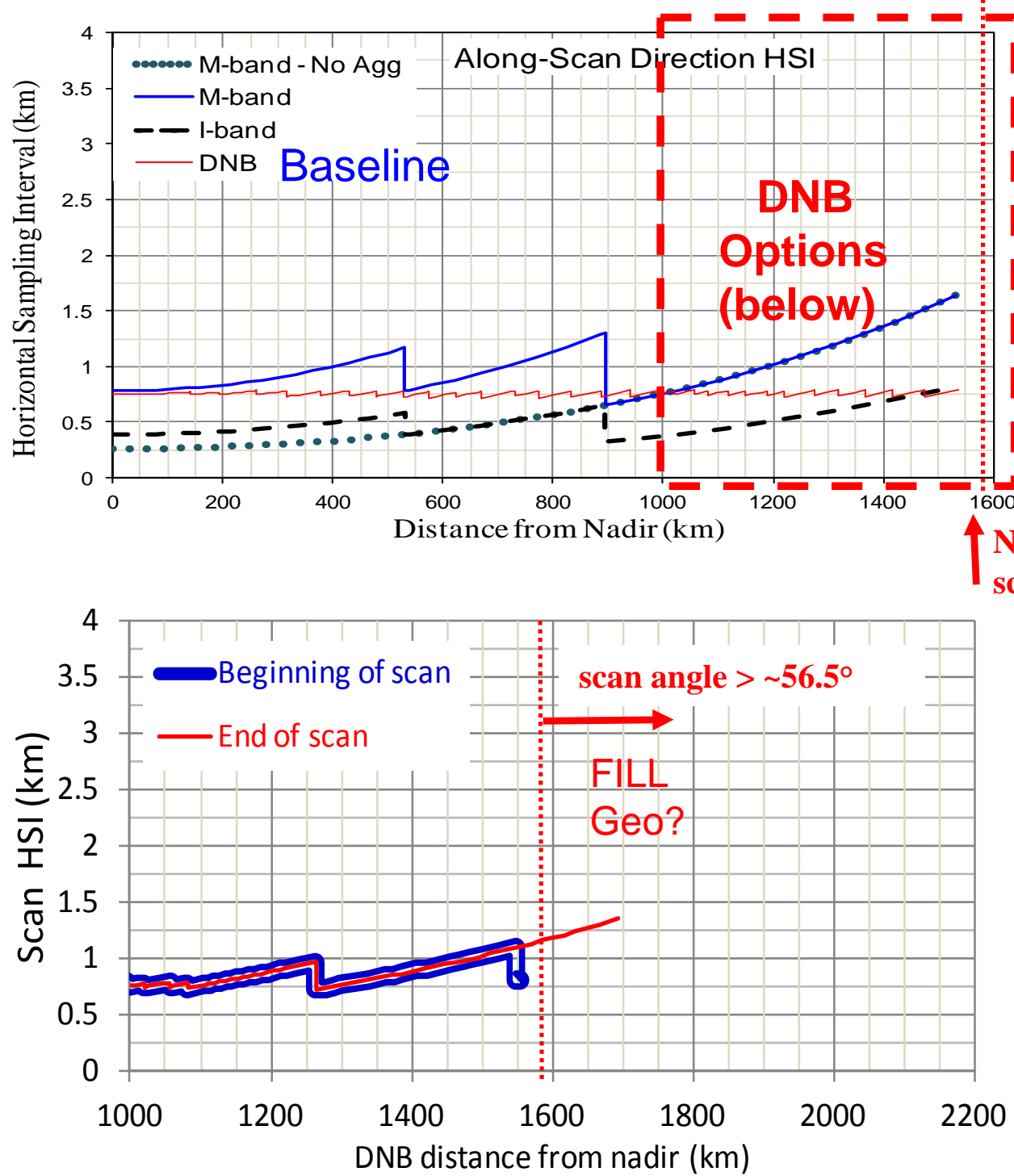

"Option21-26"
$>$ DNB LSFs are mostly square

> Baselined HSI is $\sim 750 \mathrm{~m}$

$>$ "Option21-26" has HSI max of $1.1 \mathrm{~km}$ within $56.5^{\circ}$ (up to $1.4 \mathrm{~km} @ 57.6^{\circ}$ )

$>$ "Option21" has HSI max of $1.6 \mathrm{~km}$ within $56.5^{\circ}$ (up to $3.9 \mathrm{~km} @ 60.5^{\circ}$ )

$>$ Geolocation may be FILL post-nadir for scan angle $>\sim 56.5^{\circ}$

Nominal maximum

scan angle $\left(\sim 56.5^{\circ}\right)$

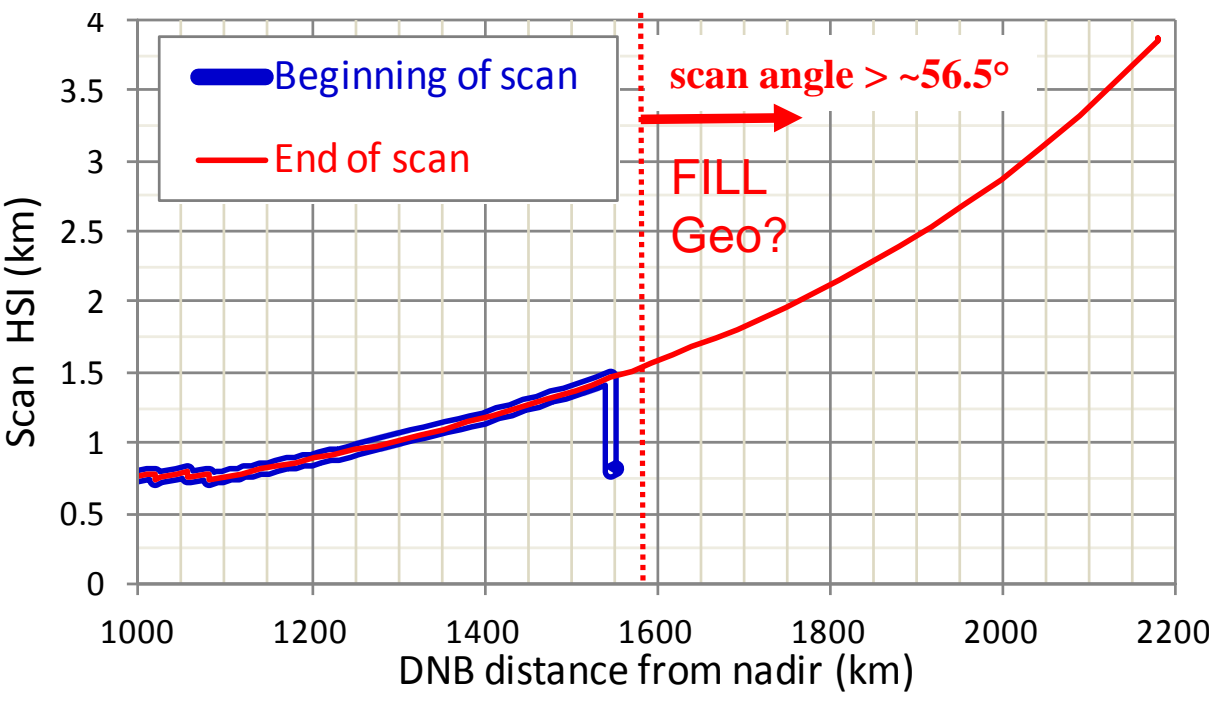

"Option21" 